\title{
Background Extraction in Electron Microscope Images of Artificial Membranes
}

\author{
A. Karathanou, J.-L. Buessler, H. Kihl, J.-P. Urban
}

MIPS laboratory, University of Haute Alsace, 4 rue des Frères Lumière, F-68093 Mulhouse Cedex, France

\begin{abstract}
On-line analysis of Transmission Electron Microscope (TEM) images is a field with great interest that opens up new prospects regarding automatic acquisitions. Presently, our work is focused on the automatic identification of artificial membranes derived from 2D protein crystallization experiments. Objects recognition at medium magnification aims to control the microscope in order to acquire interesting membranes at high magnification. A multiresolution segmentation technique has been proposed for the image partition. This paper presents an analysis of this partition to extract the background. To achieve this goal in very noisy images, it is essential to suppress false contours as they split the background into multiple regions. Statistical properties of such regions are not always sufficient for their identification as background. The analysis of these regions contours was therefore considered. In the proposed solution, the elimination of false contours is based on the statistical examination of the perpendicular gradient component along the contour. After this improved segmentation, the background extraction can be easily effectuated since this resulting region appears bright and large.
\end{abstract}

\section{Introduction}

2D crystals consist of proteins inserted within bi-lipidic layers in an organized manner. 3D information can be extracted from these artificial crystals; more specifically, it allows membrane proteins structure and function to be assessed. However, optimal conditions need to be established so that crystals formation will be correctly performed. For this purpose, a large quantity of crystallization trials is screened with a Transmission Electron Microscope (TEM). An automatic solution concerning the preparation of these trials and their image analysis is thus essential [1].

As already detailed in previous work [10], automatic assessment of 2D membranes requires the acquisition of a certain number of images at different magnifications. Each magnification step (low, medium, high) is related to two tasks: im-

Please use the following format when citing this chapter:

Karathanou, A., Buessler, J.-L., Kihl, H. and Urban, J.-P., 2009, in IFIP International Federation for Information Processing, Volume 296; Artificial Intelligence Applications and Innovations III; Eds. Iliadis, L., Vlahavas, I., Bramer, M.; (Boston: Springer), pp. 165-173. 
age acquisition, and on-line image processing. Low and medium magnification image processing aims to globally characterize the sample and determine the regions of interest to be explored. Membranes will be finally examined at high magnification to assess the success of the crystallization. We present here certain aspects of TEM image processing acquired at medium magnification (x5000).

TEM images at medium magnification appear globally within a wide gray level range as they can contain dark objects (protein or membrane aggregates, staining artifacts) within a bright background with gray level fluctuations. However, interesting non-superposed membranes are low contrasted, slightly darker than the background with borders that often mark membrane boundaries. The low contrast of the objects of interest makes image processing all the more difficult as TEM images appear particularly noisy.

The principal objective of our image analysis at medium magnification is to identify interesting membranes to provide certain statistical characteristics (such as quantity, size, shape, etc) and to trigger a new acquisition at high magnification.

This paper deals with the background-foreground objects recognition within medium magnification images. For membrane detection, a multiresolution segmentation approach has been proposed and is briefly discussed in section 2 . This segmentation algorithm leads to an image partition, without specifying which regions belong to the foreground objects or the background. A region, by itself, does not have enough characteristics to realize its classification. Membrane object identification therefore requires the background extraction. The segmentation often splits the background into smaller regions. Our approach, described in section 3, eliminates false contours that create the over segmentation. In this way, the background can be regarded as a large and bright region that can be simply extracted based on these two hypotheses.

\section{TEM Image Segmentation}

In this section we will discuss the first part of the membrane identification process: the edge segmentation algorithm applied to membrane detection.

Low contrasted membrane boundaries are not always characterized by a sufficient gradient; their gradient amplitude and/or direction varies along the contour. A multi resolution mechanism was therefore employed. Multi resolution gradient analysis, as proposed in [2], overcomes problems that common edge techniques face in TEM images. This method employs coarser resolutions to enhance edge detection. An automatic, adapted thresholding is embedded to this gradient analysis. The threshold for each resolution is determined automatically based on a histogram analysis that results in a confidence threshold of $2 \%$. In this way, at each resolution, a reasonable amount of noisy gradients is retained.

This analysis results in a set of binary images that are combined to form a reconstructed gradient like (RGL) image. The value of each pixel in the RGL image indicates the best scale at which it has been thresholded. RGL image provides a 
better compromise between edge detection and localization precision. Compared to a gradient image, the RGL image is an edge map almost noise-free and is suitable for the watershed transform.

The watershed transform is then applied on this gradient image which produces the segmented image. This algorithm progressively floods the regions starting from the minima values and marks the merging of two basins with the watershed line [3]. The resulting watershed lines situated along the edge ridges, partition the image into regions formed by 1-pixel-width closed contours; and thus providing a convenient partition of the image.

Results showed that TEM images are segmented satisfactorily. All important low contrasted membrane contours are extracted in various TEM images. We noticed that all membrane regions are detected with an over segmentation disturbing mostly the background of the image.

\section{Background Extraction}

This section deals with the second part of the membrane recognition process. After having partitioned the image into regions, we need to define the objects that are present in our images. As a straight definition for objects characteristics is difficult to be given, we can consider the recognition problem differently: including gradual steps, beginning from differentiating the background from the foreground objects by extracting it.

The problem of the background extraction is principally raised because of the absence of a simple criterion for the foreground-background separation. Common methods are based on global or local threshold techniques. However, in complex images, such methods cannot be considered. Examples of background extraction can be found in color natural images where the size, the position and the color of the background [9] are used as hypotheses for its extraction. In text document images, background is discriminated from text based on local statistical properties of predefined regions (connected components) [8]. Others refine or adapt segmentation results in order to detect the objects of interest and background [6,7].

\subsection{Background Characterization}

Region characteristics are not sufficient to classify them into backgroundforeground regions. The background is generally a large, continuous and bright region that the segmentation algorithm often splits into many unnecessary regions. Fig. 1 shows a classical example of such an over segmentation. Four of the segmented regions, where regions 1 and 4 are low-contrasted membranes and 2 and 3 background regions, present similar average intensities. This last does not allow a satisfactory discrimination between membranes and background. 

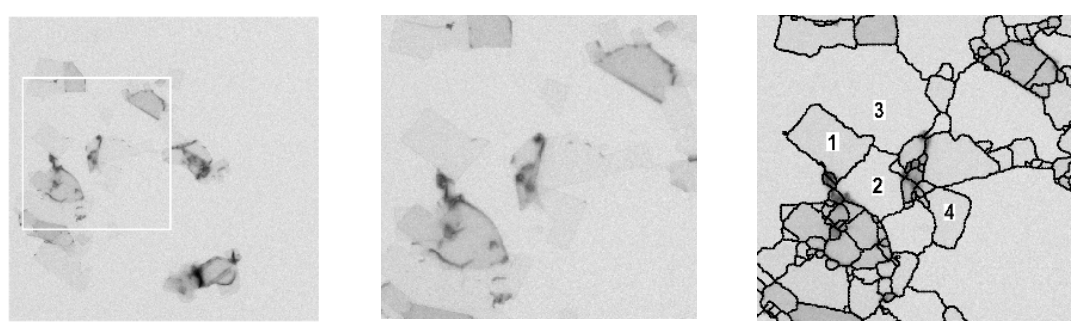

Fig. 1. Left: Initial TEM image, Center: Zoom of the white window of the initial image, Right: Segmentation of the zoomed region where, 1: membrane region with average gray level of 9180 , 2: background region with average gray level of 9250, 3: background region with average gray level of 9470, 4: membrane region with average gray level of 9310

We propose a method specific for the treatment of our images. We assume that the background region is characterized by: a) a large and continuous image region with no specific shape, b) a high average gray level, presenting sometimes important local fluctuations in combination with a strong noise, c) almost no structured gradient.

\subsection{Contour Validation}

Edge detection algorithms are disturbed by statistical fluctuations of the background. False contours appear that can be eliminated with an a posteriori validation method.

The contours, even for a low contrasted membrane, are correlated with a true gradient. This correlation is not verified with false contours, even if they have been induced by a strong gradient. The contrast in the vicinity and along the boundary is therefore clearly an important criterion.

For contour validation, we developed a method based on the gradient perpendicular to the contour [4]. The principle of this method is now described.

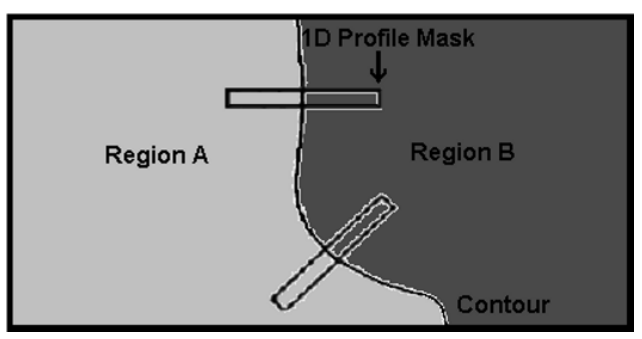

Fig. 2. Perpendicular extraction of profile transitions for a given contour segment separating regions $\mathrm{A}, \mathrm{B}$ for gradient calculation

The gradient perpendicular to the contour permits to detect and then eliminate the false contours. This gradient measure was computed for each contour pixel by 
extracting the 1D transition profile perpendicularly, as illustrated in Fig. 2, and then correlating this profile with a gradient reference filter.

The importance of noise and the assumptions concerning its nature, made statistical hypothesis test an essential step. The gradient measure is therefore assessed by means of a hypothesis test. In order to set an optimal threshold for our decision, the average correlation measure was computed for the whole segment; this last is defined as the group of edge pixels that separate two adjacent regions. This measure was reinforced by taking into account the gradient's amplitude and direction along the contour. Finally, a segment is validated if there exists a gradient perpendicular to the contour statistically significant.

However, for false contours elimination, an iterative solution was chosen as in [7]. In order to obtain meaningful regions, we searched for each one the most appropriate fusion. Results showed that this elimination is efficient concerning the background as it is not significantly disturbed by spurious contours, facilitating its extraction.

\subsection{Background Extraction Algorithm}

After false contours elimination, the background is now considered free from over segmentation. It is no more divided into small regions but appears as a large region. This characteristic is verified in all our images. This background region appears globally bright even though it presents some gray level fluctuations related to the acquisition conditions (non-uniform illumination, etc.).

We propose a background extraction technique that is composed of three steps:

1) For each region $R_{i}$ whose size is greater than a threshold $T_{s}$ :

a) Compute average gray level $G\left(R_{i}\right)$ of this region;

b) If $\mathrm{G}\left(\mathrm{R}_{\mathrm{i}}\right)$ is greater than threshold $\mathrm{T}_{\mathrm{i}}$, region $\mathrm{R}_{\mathrm{i}}$ is retained as a background region,

We introduce supplementary tests to avoid detecting a large membrane region as background, using the fact that this region neighbors the background.

2) For each background region detected, find all neighbors;

3) If two neighboring regions are selected as background, use the gradient direction of the contour segments validated during the contour validation step (section 3.2) to retain as background only the brightest region.

Thresholds $\mathrm{T}_{\mathrm{s}}$ and $\mathrm{T}_{\mathrm{i}}$ are set empirically as they are highly image dependent. As an example, $\mathrm{T}_{\mathrm{s}}$ is set to $10 \%$ of the image size, and $\mathrm{T}_{\mathrm{i}}$ to $70 \%$ of the maximal region average gray level value. These thresholds were tested for a large number of TEM images providing a satisfactory background selection. 


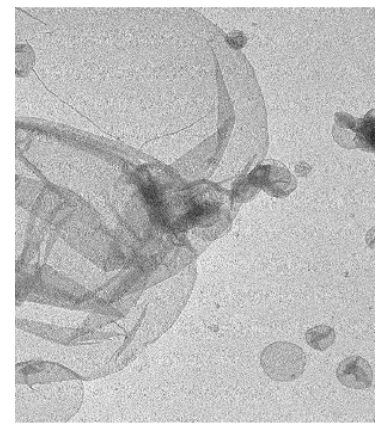

a)

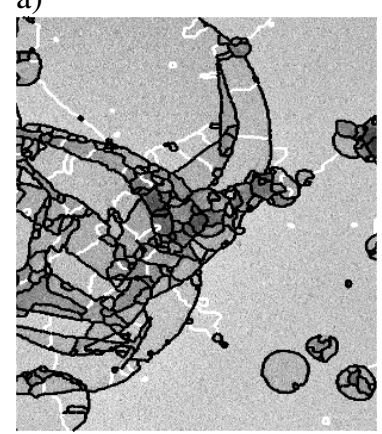

c)

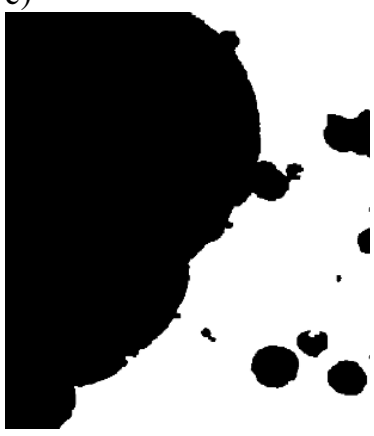

e)

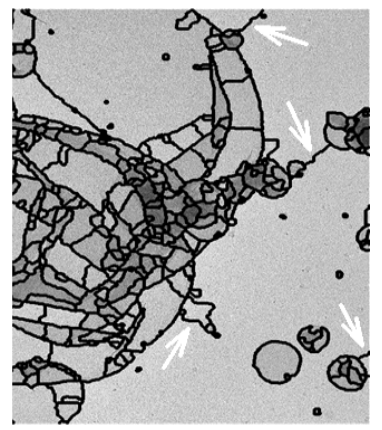

b)

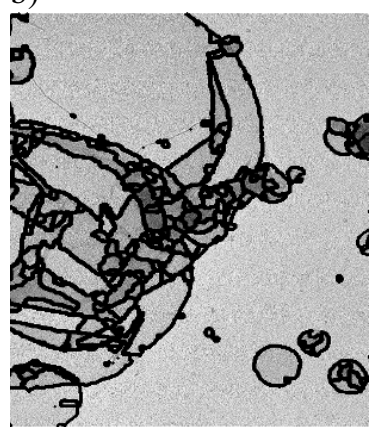

d)

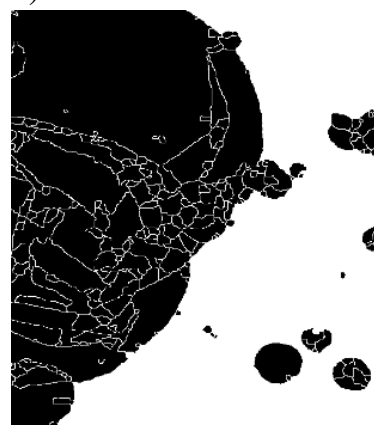

f)

Fig. 3. a) Initial image, b) Initial multiresolution segmentation with the white arrows indicating false contours over segmenting the background, c) False contour detection (in white), d) Final segmentation after elimination of false segments, e) Background extraction (in white), f) Background (in white) with segmented membrane regions (in black)

\section{Results}

The efficiency of the whole proposed method has been systematically assessed on various series of images acquired with different TEMs taken under standard acquisition conditions of illumination and exposure time. These images contain 
membranes of different types (such as sheets-like membranes or vesicles) and sizes where our algorithm was able to extract the regions of interest satisfactorily and finally identify the image background.

More specifically, the proposed segmentation and background extraction scheme has been tested on 45 representative TEM images. The quantitative evaluation of Table 1 was established according to an expert's analysis. Our technique extracted and suitably selected the important contours segmenting all foreground objects.

We consider the background well-detected even if small background regions within the image are not identified. They represent less than $4 \%$ of the total image size. Table 1 shows that the background in $87 \%$ of the images has been welldetected, among them $13 \%$ contained small undetected regions (Fig. 4). A background is considered partially detected when a background region of a more important size is not identified (representing $4 \%$ to $8 \%$ of the total image size). Complementary algorithms are currently implemented to improve the detection of this kind of regions. In the case of a background misclassification, at least $50 \%$ of the background surface is not detected.

On the other hand, foreground objects are globally properly classified as such.

Table 1. Quantitative performance measures of the background extraction algorithm for 45 representative images

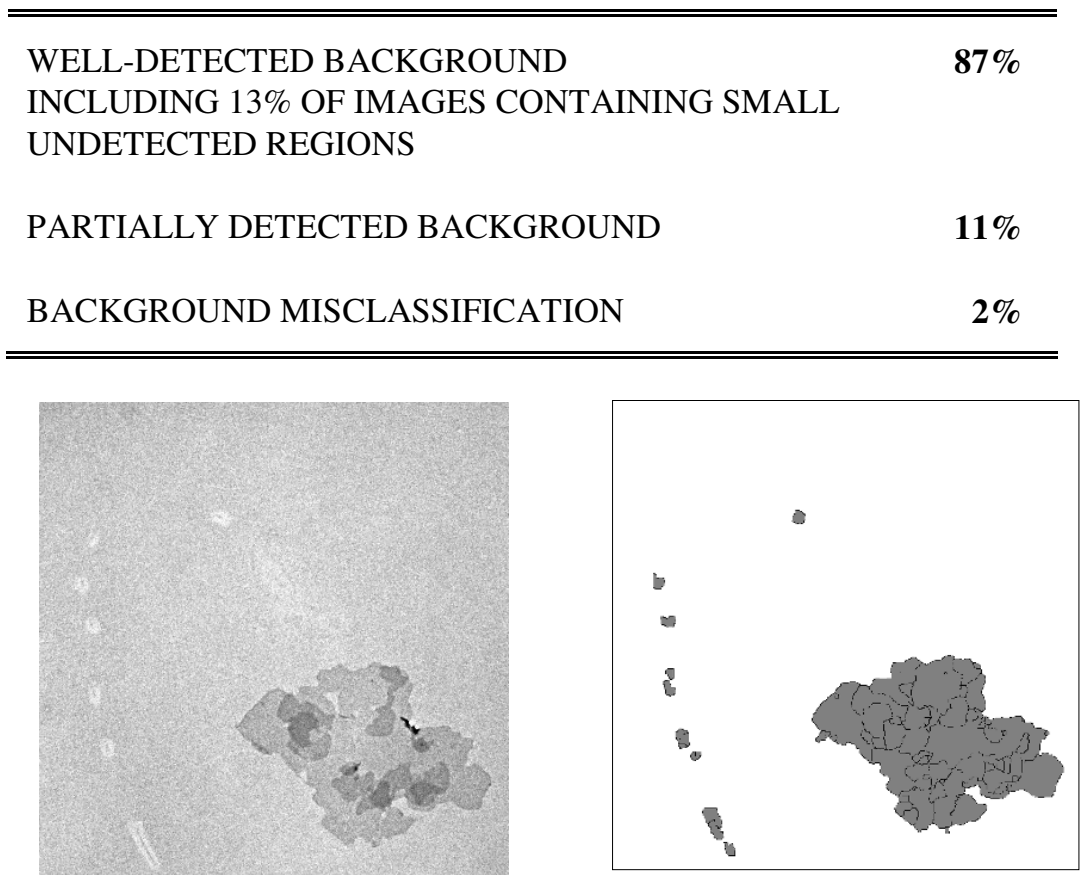

Fig. 4. Left: TEM image, Right: An example of the background extraction (in white) 
Fig.3a illustrates an example of a typical TEM image part. In the left hand side of the image, a huge vesicle stack can be observed. Fig.3b shows the initial segmentation of the image where the background over segmentation can be clearly noticed. This last is resolved by the detection and elimination of false contours as shown in the next step of the process (Fig.3c, 3d)). The background is then extracted allowing a clear distinction between the two classes objects-background.

\section{Conclusion}

We described a chain process for the extraction of the background in gray level TEM images. This process starts with a multiresolution edge extraction technique that segmented low contrasted membrane regions. False contours were then eliminated by means of a statistical validation technique. This last enables a proper false-true edge classification and therefore a correct background-object distinction. Background appears large and bright, characteristics that allow its extraction. This technique is implemented for objects recognition on electron microscope images.

Acknowledgments This work was supported by the EU 6th framework (HT3DEM, LSHG-CT2005-018811, in collaboration with the Biozentrum of Basel and FEI company who provided the TEM images.

\section{References}

1. HT3DEM: High Throughput - Three Dimensional Electron Microscopy, http://www.ht3dem.org/

2. N. Coudray, J.-L. Buessler, H. Kihl, J.-P. Urban "TEM Images of membranes: A multiresolution edge-detection approach for watershed segmentation", in Physics in Signal and Image Processing (PSIP), 2007

3. L. Vincent and P. Soille, "Watersheds in digital spaces: an efficient algorithm based on immersion simulations", IEEE Transactions on Pattern Analysis and Machine Intelligence, vol. 13, pp. 583-598, 1991

4. Karathanou, J.-L. Buessler, H. Kihl, and J.-P. Urban, "Detection of low contrasted membranes in electron microscope images: statistical contour validation", Digital Imaging Sensors and Applications, Imaging Science and Technology/SPIE, 21st Annual Symposium on Electronic Imaging, 2009.

5. Kostas Haris, Serafim N. Efstratiadis, Nicos Maglaveras and Aggelos K. Katsaggelos, "Hybrid image segmentation using watersheds and fast region merging", IEEE Transactions on Image Processing, vol. 7, pp. 1684-1699, 1998

6. Lifeng Liu and Stan Sclaro, "Shape-Guided Split and Merge of Image Regions", 4th International Workshop on Visual Form, vol. 2059, pp. 367-377, 2001

7. Theo Pavlidis and Yuh-Tay Liow, "Integrating region growing and edge detection", IEEE Transactions on Pattern Analysis and Machine Intelligence, vol. 12, pp. 225233, 1990 
8. Utpal Garain, Thierry Paquet, Laurent Heutte, "On foreground - background separation in low quality document images", International Journal on Document Analysis and Recognition, vol. 8, pp. 47-63, 2006

9. Yi Lu and Hong Guo, "Background Removal in Image indexing and Retrieval", 10th International Conference on Image Analysis and Processing, pp. 933, 1999

10. Nicolas Coudray, Jean-Luc Buessler, Hubert Kihl, Jean-Philippe Urban, "Automated image analysis for electron microscopy specimen assessment", 15th EUropean SIgnal Processing COnference (EUSIPCO), pp. 120-124, 2007 